\title{
Research and Strengthening of Sensitive Power Grid
}

\author{
Tianqi Lu $^{1, a}$, Dongwei WANG ${ }^{2, b}$, Ran Liu ${ }^{1, c}$, Jiye Yang ${ }^{1, d}$, Xun Gao ${ }^{1}$, Youyin \\ Wang $^{1}$, Xianglin $\mathrm{Li}^{1}$, Tao $\mathrm{Liu}^{1}$, Song $\mathrm{Ju}^{1}$ \\ ${ }^{1}$ State Grid Liaoning Electric Power Company Limited Economic Research Institute, Shenyang, \\ 110015, China \\ ${ }^{2}$ Karlsruhe institute of technology,Germany \\ aemail: lutianqi5558972@sina.com, bemail: ueeox@student.kit.edu \\ cemail: psincos@163.com, ${ }^{\mathrm{d}}$ email: yjy-cmy@163.com
}

Keywords: Algorithm; Converge rate; Leader

\begin{abstract}
Based on distributed algorithm, in order to make it more effective, we hope to optimize certain steps of the algorithm by the introduction of PSO. PSO is one of the swarm intelligence algorithm that has been developed in recent years aiming at multi-objective optimization, which based on the hypothesis that every particle in the swarm could acquire information from its or other particle's origin experiences[1]. PSO possess high rate of convergence, and few parameters are required. When it applied to the decision of the leader in distributed algorithm, we hope to find one or severe proper leaders more quickly. The introduction of PSO under this circumstance will make the task easy to complete, therefore enhance the effective of distributed algorithm. The paper contains the principle and mathematical formulation of standardized PSO, and present program of PSO when applying to distributed algorithm for the sake of algorithm's nature testing.
\end{abstract}

\section{Introduction}

In the context of power systems, the trend is society's increasing demand for power supply performance, power users will pay more, will encounter a more time-varying load conditions, this requires network controller can effectively and quickly react to the changes anywhere in the network [2]. In the case of total output to meet demand, minimize cost, and economic factors are taken into account on the grid controller processing power requirements [3] is in the power system network, with new ways of distributing control, as interaction networks in power system communication method to improve performance of the electric power system.

\section{Distributed Algorithm}

\section{Basic concepts of distributed algorithm and algorithm.}

Distributed algorithms are applied to systems network, distributed algorithm for network topology structure must be the basic factor for consideration. We use a simple matrix of the adjacent to describe the structure of the network, which is an $\mathrm{NxN}$ matrix. After various nodes of the network are numbered 1 to $\mathrm{n}$ and off-diagonal element represents the number of connections from node I to node $\mathrm{j}$. In undirected networks presented in this chart, this matrix is a diagonal elements is $0(0,1)$-matrices, and is symmetric.Array $\mathrm{L}=\left[l_{i j}\right]$.

In this array, $l_{i j}=\sum_{i \neq j} a_{i j}$ for the diagonal elements, $l_{i j}=-a_{i j}$ for non-diagonal elements. $\mathrm{L}$ matrix is the $n \times n$ matrix. For an undirected graph, L matrix is called the Laplacian matrix. Traditional control methods with Lagrange multipliers method to solve economic problems, based on the assumption that each motor has no power limit is reached, and each motor in the optimum operation point have the same marginal cost of IC. Consistency algorithm ensures that all variables are converging on a common set of values, thus the marginal cost of IC were selected as consistent variable [4].

Hypothesis generator unit cost of a quadratic equation: 


$$
C i(P G i)=a_{i}+b_{i} P_{G i}+C i P_{G i}^{2}
$$

Solve the economic purpose of the assignment is to make a system of $n$ generators of minimizing the total running costs:

$$
C_{\text {total }}=\sum_{i=1}^{n} C_{i}\left(P_{G i}\right)
$$

Under the power balance and electrical output capacity limitations:

$$
\begin{aligned}
& P_{D}-\sum_{i=1}^{n} P_{G i}=0 \\
& P_{G i, \min } \leq P_{G i} \leq P_{G i, \max }
\end{aligned}
$$

$P_{G i}$ represents output power of units i, $P_{D}$ represents aggregate capacity.Assuming that all generators are running their power limits.Among consistency algorithm, IC values for each motor is defined as traditional economic allocation:

$$
I C_{i}=\frac{d C_{i}\left(P_{G i}\right)}{d P_{G i}}=\lambda_{i=1,2,3 \ldots . . n}
$$

IC values described by the first-order discrete consistency algorithm is as follows:

$$
\lambda_{i}[k+1]=\sum_{j=1}^{n} d_{i j} \lambda_{i}[k]_{i=1,2,3 \ldots . . n}
$$

$d_{i j}$ is that random matrices which wasmentioned before. Compliance (6) describes the subordinate node correction law systems will be brought together in a common IC value of each node.

\section{Simulation analysis of distributed algorithms.}

Distributed computing: Figure 1 is a 15-node communication network two nodes in the example of simulation results, each time you enter the number of the selected node is obtained the result as the leading node of this node.

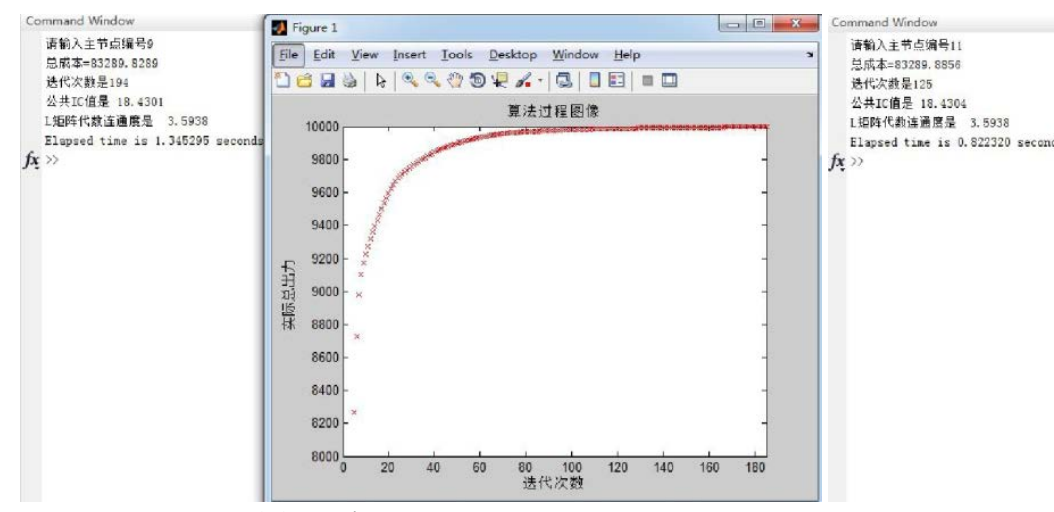

(a)node 9

Figure.1. Leading node simulation results

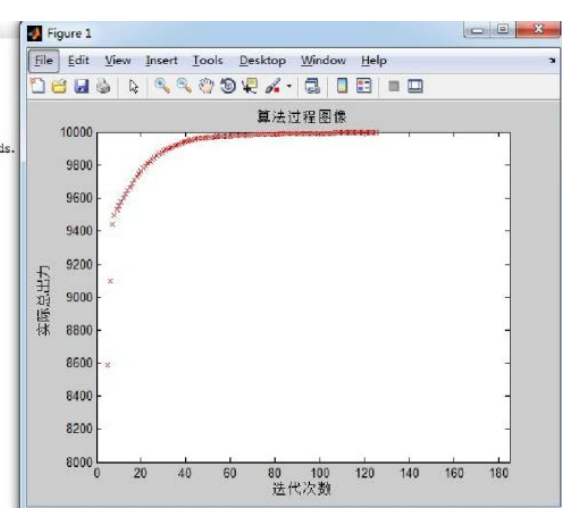

(b)node 11

\section{Particle swarm optimization}

\section{PSO basic concepts.}

Function value at which to evaluate is the Particle Swarm Optimization algorithm in Adaptive value, function is the Particle Swarm algorithm for the calculation of the fitness function; update their positions following the formula is the position of the Particle Swarm Optimization algorithm speed update formula. Under normal circumstances, we have $n$ particles on Q-dimensional spaces optimal search function, each particle is expressed as: $X_{i}=\left(X_{i 1}, X_{i 2} \ldots, X_{i Q}\right)$,Corresponds to the speed of each particle can be expressed as: $V i=\left(V_{i 1}, V_{i 2} \ldots, V_{i Q}\right)$ Speed and position of the Particle Swarm Optimization algorithm to update formula: 


$$
\begin{aligned}
& V_{i d}^{k+1}=w V_{i d}^{k+1}+C_{1} \xi\left(P_{i d}^{k}-X_{i d}^{k}\right)+C_{2} \eta\left(P_{g d}^{k}-X_{i d}^{k}\right) \\
& X_{i d}^{k+1}=X_{i d}^{k}+r X_{i d}^{k+1}
\end{aligned}
$$

$\mathrm{W}$ is keeping the original speed of the coefficients, so called inertia weight, ability to keep their original speed. Particle Swarm in the actual operation of the process, and w values can change with the iteration count, so as to ensure that at the beginning of the iteration to quickly close to optimal values close to, but also ensures sufficient precision in the latter stage to ensure that better optimal solutions. Is a coefficient of inertia weight in particle track your best value, which represents the particles on their own understanding of so called "cognitive" [5], and usually set to 2. Optimal particle tracking groups of inertia weight, optimal information perception and particles for the whole group to follow, so called "social knowledge", simple can be set to 2. , Is the [0,1] range of uniformly distributed random numbers. When $r$ is location updates, speed in front of a coefficient, called constraint factor, usually set to 1 , updates similar to the distance per unit of time.

\section{Particle Swarm algorithm}

PSO algorithm is a random, parallel optimization algorithm. Its advantages are: do not require the optimized function to have differentiable, can be guided, continuous and other properties, can be used for traditional mathematics can not solve the problem of function optimization, convergence speed, the algorithm is simple and easy to implement, from its formula can See these features, the required parameters less flow chart structure is simple. Suitable for real value processing.

However, the shortcomings of PSO algorithm are: (1) for a number of local extreme point function, easy to fall into the local extreme point, the correct results. There are two reasons for this phenomenon, one is due to the nature of the function to be optimized; the second is due to the PSO algorithm in the diversity of particles quickly disappear, resulting in premature convergence. These two factors usually work together to make the particle swarm algorithm into a local optimal, this chapter of the PSO simulation can see this point. (2) Because the search method is not sophisticated, PSO algorithm is often difficult to get accurate results, the cause of this problem is

PSO algorithm does not make full use of the information obtained in the calculation process, in each step iterations, only the use of group optimal and individual optimal information. (3) Although the PSO algorithm provides the possibility of global search, but can not guarantee convergence to the global optimal point, which is the number of iterations, the nature of the function. (4) PSO algorithm is a bionic optimization algorithm, there is no strict theoretical basis, only through a group search for a simplified simulation of the design, and not from a mathematical point of view why this algorithm is valid, and its Applicable scope. Therefore, PSO algorithm is generally applicable to high-dimensional, there are multiple local extreme points and do not need high-precision solution of the optimization problem.

\section{Distributed control using simulation and analysis of particle swarm optimizer}

On the Particle Swarm Optimization algorithm based on distributed algorithm of fitness function, the fitness function of the independent variable is the number of particles, that is different as the lead node; single target output is iterations of the corresponding node[6]. Particle Swarm Optimization as a whole aims to find minimum value, minimum number of iterations of the node number and when this node as the dominant nodes distributed when the number of iterations of the algorithm. According to the program, calculation and simulation of distributed algorithm for our (15-node communication network example) using data to test. In this calculation, the number of particles is set to 30 , the number of iterations is set to 10 : 


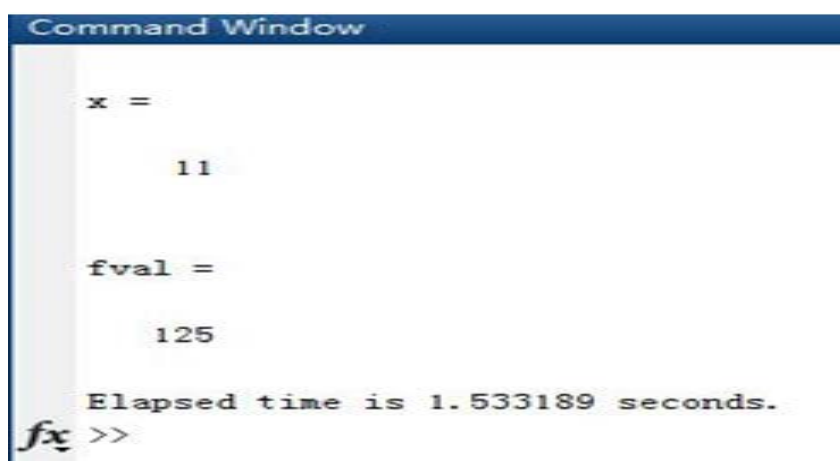

Figure.2. the number of particles is 30 , the number of iterations is 10 results

Particle Swarm Optimization gives a result soon. The results show that by 1.53 seconds, 10 iterations converges to this node number 11 all particles, and when the nodes do main nodes only 125 distributed algorithms can satisfy the accuracy requirements for the iteration. Upon inspection, when as a 15 point lead node, distributed algorithms needed to reach the preset accuracy number of iterations as shown below:

Table.1.node data

\begin{tabular}{lll}
\hline & & \\
Leading node number & Number of iterations & time cost $(\mathrm{s})$ \\
\hline 1 & & \\
2 & 199 & 0.188788 \\
3 & 168 & 0.138972 \\
4 & 223 & 0.267873 \\
5 & 169 & 0.207010 \\
6 & 231 & 0.216698 \\
7 & 185 & 0.143533 \\
8 & 139 & 0.156687 \\
9 & 184 & 0.163603 \\
10 & 194 & 0.112315 \\
11 & 239 & 0.231398 \\
12 & 125 & 0.126039 \\
13 & 138 & 0.125131 \\
14 & 338 & 0.325569 \\
15 & 168 & 0.158749 \\
\hline
\end{tabular}

As can be seen from this table, take the number 11 when a node is node-led, distributed algorithm iterations of at least 125, with Particle Swarm Optimization algorithm for optimal results are consistent. Which initially reflected a particle swarm optimization algorithm applied in distributed algorithms for reasonableness and correctness. This is cases is only 15 a node of communications network, actual of grid in the, node number more huge, with enumeration method find led node, not only node number increased, and for each node of calculation volume also will more big, led to operation total increased more quickly, enumeration method looking for best node of method certainly will increasingly slow, that cannot meet complex grid for sensitive sex of requirements. In this way, Particle Swarm Optimization algorithm in relation to simple enumeration method in looking for the best on the primary node's speed advantage will be even more obvious.

\section{Conclusion}

The distributed algorithm itself can be improved, that is, whether the choice of its dominant node will affect the performance of the distributed algorithm to a large extent. Which in itself is an easy task to find the traditional mathematical model, the emergence of evolutionary algorithms for such problems pointed out a Ming Road. This paper studies the particle swarm algorithmPSO in 1995 
and no scientists in the study of bird movement, it is relative to other evolutionary algorithms, with a fast convergence, the formula is simple, easy to implement, and only a small number of parameters need to adjust the advantages, and since the proposed, And soon became a new research hotspot in the field of evolutionary computation, which has been widely used in multi-objective optimization problem. This paper is an attempt to apply particle swarm optimization to the field of power system optimization.

\section{References}

[1] $\mathrm{Hu} \mathrm{X}$, Eberhart R C,Shi Y. Particle swarm with extended memory for multi-objective optimization[J]. Proceedings of the IEEE Swarm Intelligence Symposium, Indianapolis, Indiana, USA 2003, 193 197

[2] Gao Ying, Xie Shengli. Immune Particle Swarm Optimization algorithm [J]. Computer engineering and applications, 2004,41 (6): 4 6.

[3] Gao Ying, Xie Shengli. Particle Swarm Optimization algorithm based on simulated annealing [J] computer engineering and applications, 2004,1:47 50

[4] Chen Shaoxing, multi-objective Particle Swarm Optimization algorithm and its application research. Dalian University of technology master's thesis 2007.12.

[5] ShipingYang,Sicong Tan,and Jian-Xin Xu. Consensus Based Approach for Economic Dispatch Problem in a Smart Grid.IEEE Transactions on power systems,VOL.28, NO.4,NOVMEMBER 2013

[6] ping, Zhang yaoxue. improved Particle Swarm Optimization algorithm and its application in power system. 2012.4 South China University of technology PhD theses 